\title{
In situ observations and measurements of plastic deformation, phase transformations and fracture with 4D-STEM
}

Yang Yang ${ }^{1}$, Ruopeng Zhang ${ }^{2}$, Shiteng Zhao ${ }^{1}$, Yu Deng ${ }^{3}$, Qin Yu ${ }^{4}$, Steven Zeltmann ${ }^{1}$, Sheng Yin ${ }^{4}$, Jim Ciston $^{5}$, Colin Ophus ${ }^{6}$, Mark Asta ${ }^{1}$, Robert Ritchie ${ }^{1}$ and Andrew Minor ${ }^{7}$

${ }^{1}$ UC Berkeley, United States, ${ }^{2}$ National Center for Electron Microscopy, Molecular Foundry, Lawrence Berkeley National Laboratory, Berkeley 94720, USA, United States, ${ }^{3}$ Nanjing University, United States, ${ }^{4}$ LBNL, United States, ${ }^{5}$ UC Berkeley, California, United States, ${ }^{6}$ Lawrence Berkeley National Laboratory, California, United States, ${ }^{7}$ UC Berkeley, Berkeley, California, United States

In situ TEM experiments are typically recorded either in real space or diffraction space. However, it would be ideal to have information from both for when transient events occur that cannot be repeated exactly (iedefect generation or irreversible phase transformations). 4D-STEM can come close to providing simultaneous real-space imaging and diffraction analysis during in situ testing, making it possible to perform strain mapping via diffraction pattern analysis during in-situ deformation in a TEM. This talk will highlight recent in situ 4DSTEM nanomechanical deformation experiments that explore transient events where both information from diffraction space and real space are used. The diffraction patterns are used to identify different phases, defects, orientations and relative strain, while the images formed by using virtual apertures provide microstructural context for the analysis. Example experiments include defect generation and fracture in multi-principal element alloys [1] and phase transformations in functional oxides [2].

\section{ACKNOWLEDGEMENTS}

Primary support for this work came from the Director, Office of Science, Office of Basic Energy Sciences, Materials Sciences and Engineering Division, of the U.S. Department of Energy under Contract No. DEAC02-05-CH11231 within the Damage-Tolerance in Structural Materials (KC 13) program at the Lawrence Berkeley National Laboratory. YD acknowledges support by the National Natural Science Foundation of China (Grants Nos. 50802039) and Natural Science Foundation of Jiangsu Province, China (Grant Nos. BK20151382). R.Z. and S.Z. acknowledge funding from the US Office of Naval Research under Grant Nos. N00014-19-1-2376 andN00014-17-1-2283, respectively. supported by STROBE, A National Science Foundation Science \& Technology Center under Grant No. DMR 1548924. The authors acknowledge support from the Molecular Foundry at Lawrence Berkeley National Laboratory, which is supported by the U.S. Department of Energy under Contract No. DE-AC02-05-CH11231. J.C. and C. O. Acknowledges additional support from the Department of Energy Early Career Research Program. 

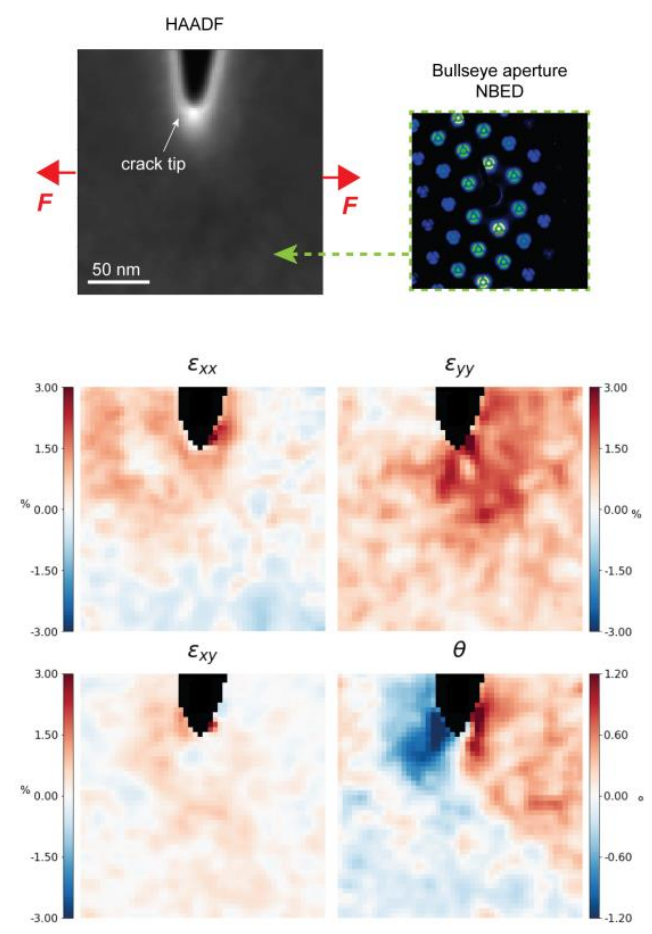

Figure 1. 4D-STEM strain mapping of crack tip in CrCoNi medium entropy alloy during in situ deformation.

\section{References}

[1] Ruopeng Zhang, Shiteng Zhao, Jun Ding, Yan Chong, Tao Jia, Colin Ophus, Mark Asta, Robert O. Ritchie \& Andrew M. Minor "Short-Range Order and Its Impact on the Properties of the CrCoNi Medium Entropy Alloy" Nature, 581 (7808), 283-287

[2] Yu Deng, Christoph Gammer, Jim Ciston, Peter Ercius, Colin Ophus, Karen Bustillo, Chengyu Song, Ruopeng Zhang, Di Wu, Youwei Du, Zhiqiang Chen, Hongliang Dong, Armen. G. Khachaturyan and Andrew M. Minor, "Hierarchically-structured superelastic deformation in ferroelastic-ferroelectrics", Acta Materialia, 181, 501-509 (2019) 